\title{
Evaluación de un programa de educación ambiental desde la voz del alumnado
}

\author{
Gabriel Prosser-Bravo, Psicólogo \\ Universidad de Chile, Chile*
}

María Soledad Salazar-Sepúlveda, Lic.

Universidad de Chile, Chile**

Sonia Pérez-Tello, Ph.D.

Università Cattolica del Sacro Cuore di Milano, Italia***

\author{
Marcela Pérez-Lienqueo, Ingeniera \\ Iplacex, Chile ${ }^{\star \star \star \star}$
}

Carlos Prosser-González, Consultor

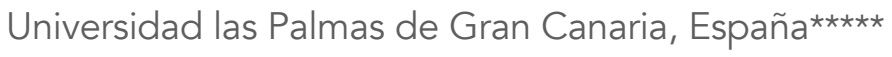

gabrielprosserb@gmail.com

\section{Resumen (analítico)}

Los niños, niñas y adolescentes no participan activamente de los sistemas, proyectos y políticas ambientales que se implementan, lo que supone una vulneración de derechos y un erreur fatale para la retroalimentación de estas intervenciones. La presente investigación describe los resultados de una evaluación cualitativa realizada a un programa de educación ambiental local, mediante asambleas infantiles en las que participaron 150 escolares de entre 6 y 14 años. Estos y estas fueron capaces de detectar ciertas dificultades y activos del programa, algunos vacíos y buenas prácticas presentes en la escuela, así como también mencionaron una serie de problemáticas socioambientales tanto locales como globales. Se concluye que estas personas son totalmente válidas para participar en la evaluación de programas sociales; así mismo, la necesidad de incluir sus opiniones en la mejora de los proyectos ambientales.

\section{Palabras clave}

Participación infantil, evaluación de la educación, educación ambiental, niñez, adolescencia.

\section{Thesauro}

Tesauro de Ciencias Sociales de la Unesco.

\section{Para citar este artículo}

Prosser-Bravo, G., Salazar-Sepúlveda, M. S., PérezTello, S., Pérez-Lienqueo, M., \& Prosser-González, C. (2020). Evaluación de un programa de educación ambiental desde la voz del alumnado. Revista Latinoamericana de Ciencias Sociales, Niñez y Juventud, 18(2), 1-26. http://dx.doi.org/ 10.11600/1692715x.18206

Historial

Recibido: 14.12 .2019

Aceptado: 09.02.2020

Publicado: 15.05 .2020

\section{Información artículo}

Este artículo se deriva de una evaluación municipal multinivel realizada al Programa de Innovación Pedagógica y Educación para la Sustentabilidad de la Municipalidad de Los Ángeles, Chile. El proyecto contó con financiamiento autogestionado y fue realizado entre mayo de 2018 y abril de 2019. Área: Ciencias sociales. Subárea: Temas especiales. 


\title{
Evaluation of an environmental education program through the voices of students
}

Abstract (analytical)

Children and adolescents do not actively participate in environmental systems, projects and policies that are implemented, which implies a violation of their rights and a fatal mistake in the evaluations of these interventions. This research describes the results of a qualitative assessment of a local environmental education program through Children's Assemblies that involved the participation of 150 students between 6 and 14 years of age. These children were able to identify certain difficulties with the components of the program, gaps and best practices in their schools and a series of local and global socio-environmental problems. The authors conclude that these children are fully valid participants in the evaluation of social programs while identifying the need to include their opinions in the improvement of environmental projects.

Keywords

Child participation, educational evaluation, environmental education, childhood, adolescence.

\section{Avaliação de um programa de educação ambiental desde a voz dos alunos}

\author{
Resumo (analítico)
}

Crianças e adolescentes não participam ativamente dos sistemas, projetos e políticas ambientais implementados, o que implica em violação de direitos e erro fatal para o feedback dessas intervenções. Esta pesquisa descreve os resultados de uma avaliação qualitativa realizada em um programa local de educação ambiental, por meio de assembleias infantis, nas quais participaram 150 escolares de 6 a 14 anos. Estes foram capazes de detectar certas dificuldades e ativos do programa, algumas lacunas e boas práticas presentes na escola, e mencionaram uma série de problemas socioambientais tanto locais como globais. Conclui-se que esses temas são plenamente válidos para participar da avaliação de programas sociais, bem como a necessidade de incluir suas opiniões na melhoria de projetos ambientais.

\section{Palavras-chave}

Participação infantil, avaliação educacional, educação ambiental, infância, adolescência.

Información autoras

[] Psicólogo de la Universidad de Chile. Diplomado en Psicología Social Crítica de la Universidad de Chile. ID 0000-0003-1255-5890. Correo electrónico: gabrielprosserb@gmail.com

[**] Licenciada en Psicología de la Universidad de Chile. (iD 0000-0002-0354-2740. Correo electrónico: sole5013@gmail.com

[***] Psicóloga de la Universidad de Chile. Master en Psicología escolar Instituto Carlo Amore- Centro di Studi Bruner (Italia). Doctora en Psicología Social y del Desarrollo de la Università Cattolica del Sacro Cuore di Milano (Italia). iD 0000-0001-7867-6447. Indice H5: 1. Correo electrónico: sonperez@u.uchile.cl

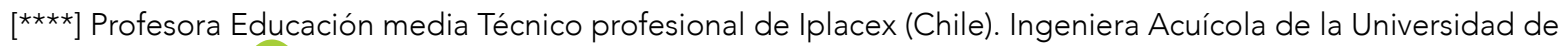
los Lagos (Chile). iD 0000-0001-6294-294X. Correo electrónico: mperez@educacionlosangeles.cl

[****] Consultor ambiental de la Universidad las Palmas de Gran Canaria (España). ID 0000-0001-7938-4171.

Correo electrónico: prossercarlos@gmail.com 


\section{Introducción}

E 1 problema del calentamiento global es cada vez más grande. Para el año 2100 la ras a nivel económico, político, geográfico y social (Dixson-Declève \& Wijkman, 2019). Pese a estar enfrentados a la mayor amenaza de la historia de nuestra especie, los planes de respuesta han sido deficientes, siendo los principales poderes mundiales los responsables de obstaculizar una acción colectiva que logre los objetivos propuestos (World Economic Forum, 2019).

Ante este escenario, los gobiernos locales han promulgado una serie de medidas para promover el desarrollo de capacidades proambientales en una ciudadanía capaz de construir un mundo sustentable y justo socialmente (González \& Bonan, 2017). Dentro de estas se encuentran los programas de educación ambiental (PEA), orientados a múltiples edades que pueden darse, o no, en contextos institucionales e intentan promover acciones en pos de la ecociudadanía (González-Gaudiano \& Maldonado-González, 2017).

Pese al probado efecto de estos proyectos sobre el nivel individual, escolar y comunitario (Espejel \& Flores, 2017; Olsson et al., 2016; Olsson et al., 2019), la mayoría de sus evaluaciones no considera la opinión de sus participantes, lo que desatiende las necesidades, intereses y posibles aportes que puedan surgir de quienes están en primera línea (Mogren et al., 2018; Peters et al., 2013).

Este descuido sería un error más grave en aquellos PEA que se dan en contexto escolar, ya que sus características presentan una serie de dificultades y desafíos particulares (Saidón \& Claverie, 2016). Dada la falta de investigaciones al respecto, existe la necesidad de evaluar las experiencias educativo-ambientales desde sus diversos actores para una mejor retroalimentación (Medina \& Páramo, 2014; Muñoz-Montilla \& Páramo-Bernal, 2018). En especial, cuando la participación en programas educativos no es igual entre niños, niñas y adolescentes (en adelante NNA) y los integrantes adultos (Ceballos- 
López et al., 2019; Susinos-Rada \& Ceballos-López, 2012), lo que permite presuponer que tal situación se repite en la educación ambiental (Mackey, 2012).

En busca de cuestionar lo anterior, este trabajo describe la evaluación realizada a un programa escolar de educación ambiental local el cual se desarrolla en 51 establecimientos educacionales al sur de Chile. Ello se hizo mediante asambleas infantiles con participación consultiva de 150 NNA de entre 6 a 14 años que forman parte del programa. Todo producto de una evaluación municipal que abrió las puertas para que los y las escolares fueran protagonistas de la elaboración de futuras versiones del programa.

\section{Participación infantil y voz del alumnado}

Hablar de conceptos como participación infantil y voz del alumnado propone visibilizar un problema pedagógico y político que pasa frecuentemente inadvertido (SusinosRada \& Ceballos-López, 2012). Desde esta posición, es importante promover estrategias para la participación e involucramiento de los menores en sus proyectos educativos y de vida, para así implicarles en su formación y en su capacidad para actuar colectivamente (Fielding, 2011).

Pese al interés en los últimos 50 años, este campo se encuentra aún en una «temprana edad» (Contreras \& Pérez, 2011; Liebel, 2007; Susinos-Rada \& Ceballos-López, 2012; Voltarelli, 2018), aun cuando, para muchos especialistas, la participación de estos sujetos supone una transformación de la relación educador-educando, del ámbito curricular y de temas relacionados con la gestión de la escuela y de la vida sociocomunitaria (Díaz, 2010; Díaz-Bórquez, Contreras-Shats, \& Bozo-Carrillo, 2018; Rudduck \& Flutter, 2007).

En este marco, las categorías de participación de los y las escolares han variado según el acento en: la relación con el docente, el lugar de los infantes en la comunidad escolar o el grado de participación en los proyectos intra y extra escolares (Fielding, 2011; Fielding \& McGregor, 2005; Trilla \& Novella, 2001). Una de las clasificaciones más notada es la ofrecida por Hart (2013), quien sitúa en el grado menor aquello que no sería participación: la manipulación, la decoración y la participación simbólica. En un segundo grado distingue otros tres niveles: cuando los NNA son asignados e informados, cuando son consultados e informados y aquellos proyectos iniciados por adultos, pero de decisiones compartidas. Por último, aparecen los dos niveles de mayor participación: aquellos movilizados solo por menores y, los que siendo iniciados por estos, permiten participar a todos. 
Aun cuando la voz del alumnado es citada por algunos autores clásicos desde hace años (Mitra, 2007; Susinos-Rada \& Ceballos-López, 2012; Trilla \& Novella, 2001), el tema de la evaluación ha sido apenas desarrollado en español, siendo escasas las evaluaciones de estudiantes sobre su escuela o sobre programas que aborden problemáticas sociales que les circundan (Ceballos-López \& García-Becerra, 2018; Ceballos-López et al., 2019).

En específico, recientes investigaciones señalan que la evaluación es un aspecto de la educación en el que se tiende a dejar fuera a los niños y niñas (Hidalgo \& Perines, 2018), reproduciéndose la ideología de la inmadurez; se aduce que «aún no pueden»o «aún no deben» participar de este aspecto de la vida adulta (Díaz, 2010; Rudduck \& Flutter, 2007). Los NNA serían sujetos del futuro y no del presente, siendo importante su opinión «para el día de mañana» y no así para hoy. Son futuros ciudadanos que deben cumplir normas y deberes que, desde el exterior, se establecen sin cuestionarse profundamente el rol que puedan desempeñar en la sociedad (Ceballos-López et al., 2019).

\section{Participación infantil y voz del alumnado en la educación ambiental}

Pese a que el origen de la educación ambiental (EA) está ampliamente asociado a la Conferencia de Estocolmo (1972), no es hasta la Conferencia de Río de Janeiro (1992) que se reconoce a los niños, niñas y jóvenes como sujetos sociales interesados y con el derecho a participar de iniciativas ambientales. Esto se refleja parcialmente en la promulgación de las Agendas 21; estas, en principio, debían propiciar ciertos mecanismos participativos para los niños y las niñas, los y las jóvenes.

Hoy es difícil sostener que tales objetivos se hayan cumplido, siendo una tarea pendiente el vincularles al desarrollo de los PEA (Pramling-Samuelsson, 2011). Aun así, la falta de investigaciones de participación infantil en los PEA convive con un creciente interés académico y político por enfatizar la particularidad de trabajar la EA desde una posición que cuestione la relación educador-educando y que permita fortalecer la eco-

${ }^{1}$ Para mayor información sobre las Agendas 21 visitar la página de las Naciones Unidas https://sustainabledevelopment.un.org/outcomedocuments/agenda21 
ciudadaníaª (Ärlemalm-Hagsér, 2013; Mackey, 2012). ${ }^{3}$ A su vez, el rol de la infancia y la juventud ha sido fuertemente relevado por movimientos sociales como Friday's for Future, Youth for Climate o Yo respiro Monterrey, así como por figuras como Greta Thunberg, Emma Lim, Bruno Rodríguez o Ángela Valenzuela, quienes enfatizan su deseo de ser protagonistas de la denuncia de las ineficientes medidas gubernamentales, a la vez que lideran acciones y manifestaciones climáticas como las evidenciadas en los últimos tres años (Liebel \& Gaitán, 2019).

Según autores como Jensen (2004) o Huckle (1991), parte de este problema se debe a la tendencia general de centrarse en la transferencia de información sobre las problemáticas ambientales. Esto ha restado capacidad a los y las estudiantes para indagar sobre los problemas ambientales particulares de su territorio y centrarse en la acción, la pertinencia contextual, la participación y la interdisciplinariedad (Ceballos-López et al., 2019; Jensen, 2004). No obstante, García y Moreno (2015) en España constataron la capacidad de los NNA de vincular las problemáticas ambientales locales a los efectos globales y de detectar problemáticas tanto mundiales como de su territorio.

En Córdoba, Argentina, Imhoff y Brussino (2013) identificaron que las actividades ambientales son uno de los espacios donde más participan los y las estudiantes, aunque limitándose estas a actividades de expresión o de conducta proambiental. Lamentablemente, los grados de participación detectados descendieron ostensiblemente una vez utilizados como filtro los niveles establecidos por Hart (2013); ello dado que muchas de las actividades participativas no lo eran realmente, descendiendo la participación del 89\% al 53.5\% (Imhoff \& Brussino, 2013). Así, la relevancia de los y las niñas en la EA es innegable, pero su peso relativo en las evaluaciones y formulaciones de los PEA ha sido resistido, desatendiendo a las necesidades de participación de todos y todas ante la actual crisis ambiental.

2 El concepto proviene de los trabajos de Lucie Sauvé y otros académicos, quienes lo definen como «una forma de relación con el mundo centrada en el "vivir aquí juntos", una relación contextualizada y ubicada, que implica la responsabilidad colectiva respecto a los sistemas de vida de los cuales formamos parte y que necesita competencias para insertarse de manera eficaz en las dinámicas políticas de decisión y acción relativas a los asuntos socioecológicos» (Sauvé, 2014, p. 14).

${ }^{3}$ Este reto ha sido motor del trabajo de investigadoras que han problematizado la necesidad de trabajar la EA con menores, atendiendo las especiales características de la labor (véase Ärlemalm-Hagsér \& Davis, 2014) y formulando un campo centrado en la primera infancia, lo que ha tenido buena recibida en países angloparlantes y nórdicos (véase Hedefalk, Almqvist, \& Östman, 2015). Este espacio disciplinar no ha generado la misma atención en Iberoamérica, siendo difícil rastrear similares esfuerzos en español (véase Martínez-Agut et al., 2014). 
La no inclusión de la infancia en las evaluaciones de dichos programas supone una injusticia social, en cuanto priva del derecho de participación y opinión consignado en el artículo 12 de la Convención sobre los Derechos del Niño y resta parte importante de la sociedad que puede retroalimentar, ya que está en contacto directo con las intervenciones (Peters et al., 2013). Evaluaciones de los PEA que consideren la voz del alumnado se verán entonces favorecidas tanto por la fuente directa de información como por el lenguaje práctico y claro con el que los NNA se expresan (Escobedo et al., 2017).

El estudio que reporta este artículo surge de la evaluación de un PEA local desarrollado en Chile desde la voz de sus escolares, respondiendo a las preguntas: ¿cómo perciben el Programa de Innovación Pedagógica y Educación para la Sustentabilidad los NNA que participan de él, en una ciudad del sur de Chile?, y ¿qué propuestas de mejora le realizan? Estas preguntas suponen que la experiencia de participación en el programa generaría no solo opiniones de los y las estudiantes, sino percepciones que interpretan lo vivido en el programa, siendo así capaces de evaluar aspectos tanto internos a este como a sus condiciones de implementación. La relevancia de relevar estas percepciones está en su capacidad de retroalimentación a los programas en términos de mejora y en pos de respetar el derecho de los NNA (Ärlemalm-Hagsér, 2013; Mackey, 2012).

\section{Metodología}

\section{Descripción del caso de estudio}

El Programa de Innovación Pedagógica y Educación para la Sustentabilidad (Pipes), creado en el año 2014 en la comuna de Los Ángeles, Región del Bio-Bío, Chile, se encuentra a cargo de la Dirección de Administración de Educación Municipal. Se realiza en 51 establecimientos educacionales de la comuna, atendiendo a 12500 estudiantes y 830 docentes, estimando su población participante indirecta en 65000 personas. ${ }^{4}$ Tiene por objetivo el

integrar en su acervo curricular y transmitir en su cultura educativa, conocimientos y conceptos actuales de cuidado y protección del medioambiente; fortalecer el respeto y

\footnotetext{
${ }^{4}$ Cabe señalar que buena parte de la población de la comuna participa de manera activa en dos actividades masivas al año, más dos o tres que se realizan dentro de las escuelas o liceos (Día de la Tierra, Día del agua, WeTripantu o año nuevo mapuche).
} 
preservación de todo aquello que conforma el patrimonio de orígenes, natural y ecológico, de la comuna de Los Ángeles. (Alonso, 2018, p. 1) ( $^{5}$

El Pipes tiene una serie de líneas de acción: visitas técnicas para el desarrollo de unidades de producción vegetal; la instalación de un sistema de gestión de residuos en la escuela; acciones formativas para la comunidad escolar; formación constante y reuniones de coordinación, gestión y diseño de los docentes coordinadores y de los directivos.

La vigente Ordenanza Ambiental Comunal plantea que el programa debe orientarse a «que los estudiantes de los establecimientos educacionales municipales participen y apoyen la gestión ambiental local» (Municipalidad de Los Ángeles, 2016, p. 4). Un 15.18 \% de los docentes de la comuna está capacitado en temas ambientales y más del 10 \% de los y las estudiantes forman parte de un taller ambiental de manera permanente. Hoy se está trabajando en la instalación de un módulo transversal al plan de jornada escolar completa.

\section{Participantes}

La municipalidad ofreció a las escuelas y liceos un taller para evaluar el Pipes junto con los y las estudiantes. Accedieron voluntariamente seis establecimientos, participando 150 menores de entre 6 a 14 años, distribuidos heterogéneamente (tabla 1).

\section{Tabla 1}

\section{Datos de los participantes}

\begin{tabular}{cccccc}
\hline NE & \multicolumn{1}{c}{ Establecimiento } & N & Mujeres & $\underline{x}$ por curso & Ruralidad \\
\hline E1 & Escuela República de Alemania & 23 & $47.61 \%$ & 30 & urbana \\
E2 & Escuela la Quebrada & 26 & $58.33 \%$ & 8 & rural \\
E3 & Escuela el Nogal & 30 & $53.33 \%$ & 20 & rural \\
E4 & Escuela Nieves Vásquez Palacios & 22 & $54.54 \%$ & 25 & urbana \\
E5 & Escuela Thomas Jefferson & 32 & $59.37 \%$ & 30 & urbana \\
E6 & Liceo Santa Fe & 17 & $47.05 \%$ & 24 & rural \\
\hline
\end{tabular}

Nota: NE: número de escuela, identificación para las citas de los resultados.

\footnotetext{
${ }^{5}$ Es implementado por dos profesionales de la municipalidad que reciben apoyo de consultores externos de universidades e institutos técnicos. Centran su trabajo en el logro del Sistema Nacional de Certificación Ambiental de Establecimientos Educacionales (SNCAE), en apoyar en la obtención de fondos ambientales como el Fondo de Protección Ambiental (FPA), en la capacitación en innovación pedagógica y en la realización de actividades medioambientales comunitarias. La implementación del programa en cada escuela recae en un docente coordinador contratado para la temática ambiental. Para mayor información sobre el SNCAE visitar la página del Ministerio del Medioambiente https://mma.gob.cl/tag/sncae/ Para mayor información sobre el FPA visitar la página del Ministerio del Medioambiente http://www.fpa.mma.gob.cl
} 


\section{Técnicas de producción de información}

Se realizaron idénticos talleres en los seis establecimientos, adaptando cada uno de ellos al espacio, tamaño del grupo y recursos presentes. Los talleres tuvieron una duración de tres horas y fueron realizados en el bloque horario donde generalmente discuten y trabajan la temática ambiental en las escuelas. Constaron de tres fases: 1) detectaron de manera individual y luego en asambleas infantiles aquellos aspectos positivos y negativos del desarrollo del Pipes en la escuela; 2) jugaron una versión especial del juego piedra, papel y tijera, el cual fue adaptado a la cadena trófica de la zona; 3) escribieron una carta para una mejor educación ambiental. La primera parte del taller es la que fue analizada en el presente estudio.

Primero, se les solicitó a los NNA que pensaran individualmente un aspecto positivo y uno negativo del desarrollo del Pipes en su escuela. Posteriormente, se les agrupó de 6 a 8 menores y se les solicitó que una persona cumpliera la función de anotar los principales acuerdos. Se buscó facilitar su opinión combinando escritura, juego, movimiento, etc. (Clark, 2011). Se acogieron también las medidas de proporción sugeridas por Escobar y Bonilla-Jiménez (2017) para la conformación de los grupos, con el fin de mitigar la invisibilización de ciertas voces. Se recogió la información mediante observaciones, notas de campo y por medio de las propias actas escritas por los y las estudiantes.

Usando la taxonomía de Hart (2013), la actividad alcanzó el nivel de participación consultados e informados, dado que los NNA comprendieron el objetivo del proyecto, entendieron que su rol estaba diseñado por adultos y accedieron de manera voluntaria a hacer de asesores/consultores para dar sus opiniones y propuestas. Desde la visión de Trilla y Novella-Cámara (2001), se empleó una participación consultiva del alumnado en su subclase más participativa (similar a los consejos ciudadanos), haciendo uso de sus impresiones para valorizar lo hasta ese momento realizado y delinear proyecciones.

\section{Técnicas de análisis de la información}

Se realizó un análisis de contenido temático en dos fases: 1) se generó un pool de categorías teniendo en consideración el Sistema Nacional de Certificación de Establecimientos Educacionales, el programa Mano Verde de arborización nativa y otras evaluaciones hechas al Pipes; 2) se analizó en profundidad aquellas categorías más relevantes (Patton, 1990; Vaismoradi et al., 2013). Como medida de confiabilidad se tuvo el consenso entre los autores sobre la descripción y análisis de las categorías y subcategorías. Se tuvieron consideraciones éticas correspondientes a la investigación con comunidades y con me- 
nores como pedir la autorización a las autoridades y adultos componentes, explicar de manera clara las intenciones y voluntariedad del taller, así como el abrir un espacio extenso para las dudas y sugerencias de los NNA (Navas et al., 2018; Winkler et al., 2014).

\section{Resultados}

Cinco categorías fueron identificadas a partir del análisis de contenido: 1) dificultades en el logro de los objetivos; 2) activos del programa; 3) vacíos; 4) buenas prácticas; $\mathrm{y}$, 5) problemáticas socioambientales. Algunos de los señalamientos fueron vinculados directamente al programa, otros a la escuela y unos pocos a la sociedad, intentando distinguir con esto el locus de los problemas u oportunidades que perciben los y las estudiantes.

\section{Dificultades en el logro de los objetivos}

Se agruparon aquí los aspectos distinguidos por los NNA en los que no se ha logrado -o tan solo de manera parcial— alguno de los objetivos del Pipes. Estas debilidades no son propias de las escuelas o de los individuos; pertenecen más bien al programa y a su desarrollo, por lo que refieren a un estado incipiente en el proceso de evolución de ciertas de sus acciones. Se distinguieron cuatro subcategorías: bajo compromiso de la comunidad escolar, insuficiente desarrollo de comportamientos proambientales, sistema de gestión de residuos insuficiente o inexistente y unidad de producción vegetal deficitaria (tabla 2).

El bajo compromiso de la comunidad escolar refiere a aquellos casos en donde el programa no se difunde entre sus actores, siendo difícil que participen de manera activa. Los y las NNA identifican motivos como la falta de interés o el que a sus compañeros no les gusta participar de las actividades ambientales. Precisan también el que la participación es baja sobre todo en actividades propuestas por el programa como botar la basura, cuidar las plantas o que «falta más limpieza entre los alumnos» (G2, E2).

Cuando se habla de insuficiencia en el desarrollo del comportamiento ambiental se refiere a aquellas situaciones donde el programa no logra promover los cambios de una serie de conductas en los estudiantes y profesores. Tal como señala un estudiante: «Falta que los niños(as) se tomen en serio el taller de medioambiente» (G1, E5). Esto repercute en la falta de know-how por parte de algunas personas en acciones ambientales particulares, tales como el reciclaje o el manejo del punto limpio de la escuela. 


\section{Tabla 2}

\section{Dificultades identificadas por niños, niñas y adolescentes}

\begin{tabular}{|c|c|c|}
\hline Subcategoría & Definición & Otros ejemplos \\
\hline $\begin{array}{l}\text { Bajo compromiso de la } \\
\text { comunidad escolar (botar } \\
\text { basura y punto limpio) }\end{array}$ & $\begin{array}{l}\text { El programa logra un escaso nivel } \\
\text { de compromiso e involucramiento } \\
\text { en la comunidad escolar }\end{array}$ & $\begin{array}{l}\text { «Hay niños que no les gusta participar } \\
\text { en las actividades relacionadas con el } \\
\text { medioambiente» (G4, E1) }\end{array}$ \\
\hline \multirow{2}{*}{$\begin{array}{l}\text { Insuficiente desarrollo de } \\
\text { competencias proambien- } \\
\text { tales }\end{array}$} & \multirow{2}{*}{$\begin{array}{l}\text { El programa no logra formar en la } \\
\text { comunidad escolar las competen- } \\
\text { cias proambientales }\end{array}$} & $\begin{array}{l}\text { «Algunos niños no aprenden a } \\
\text { reciclar» }(G 1, E 2)\end{array}$ \\
\hline & & $\begin{array}{l}\text { «Los niños no utilizan de manera } \\
\text { adecuada el punto limpio» (G1, E2) }\end{array}$ \\
\hline \multirow{2}{*}{$\begin{array}{l}\text { Sistema de gestión de } \\
\text { residuos no instalado o } \\
\text { insuficiente (basureros, } \\
\text { reciclaje) }\end{array}$} & \multirow{2}{*}{$\begin{array}{l}\text { No se encuentra en el estable- } \\
\text { cimiento un sistema de gestión de } \\
\text { residuos diferente al tradicional o } \\
\text { este no logra dinamizar el proceso } \\
\text { de manera adecuada }\end{array}$} & «Necesitamos reciclar» (G1, E3) \\
\hline & & $\begin{array}{l}\text { «Lo malo es que hay pocos basureros } \\
\text { en toda la escuela» }(G 2, E 6)\end{array}$ \\
\hline & $\begin{array}{l}\text { El establecimiento no realiza la } \\
\text { plantación de árboles nativos en }\end{array}$ & \\
\hline $\begin{array}{l}\text { Unidad de producción } \\
\text { vegetal deficitaria }\end{array}$ & $\begin{array}{l}\text { sus dependencias o las unidades } \\
\text { de producción vegetal no logran su } \\
\text { pleno desarrollo debido a la mala } \\
\text { gestión de estas }\end{array}$ & «Las plantas se secan» $(\mathrm{G} 3, \mathrm{E} 3)$ \\
\hline
\end{tabular}

Otra debilidad mencionada por los NNA es la inexistencia de un sistema de gestión de residuos diferente al tradicional o su insuficiente instalación. Esto se refleja, según los y las estudiantes, en que «hay mucha basura en el suelo» $\left(G_{3}, E_{3}\right)$, una baja capacidad de reciclaje y una falta de contenedores apropiados para el número de personas del establecimiento.

La última debilidad que se identificó fueron aquellas unidades de producción vegetal desarrolladas de manera deficitaria, refiriendo a aquellos casos donde el desarrollo vegetal no es el indicado o donde, producto de la gestión humana, los resultados productivos y estéticos no son los deseados. Aquí los estudiantes señalan que hace falta una mayor arborización nativa y que, producto de un deficiente sistema de regadío, las plantas terminan por secarse, como se observa en las citas: «Que las escuelas no cuiden los árboles» $\left(\mathrm{G}_{2}, \mathrm{E}_{4}\right)$; «que no se hayan sembrado árboles nativos» $\left(\mathrm{G}_{2}, \mathrm{E}_{5}\right)$.

\section{Activos del programa}

Se utilizó el concepto activos para identificar aspectos que el Pipes aporta al logro de sus propios objetivos. Estos provienen de los componentes del programa y de otras fuentes como la municipalidad o el Estado. Se tuvo como referencia aquellos elementos del 
programa detectados en los informes técnicos, así como también aquellos provenientes de los programas que enmarcan al Pipes, detectando un total de cinco activos (tabla 3).

\section{Tabla 3 \\ Activos identificados por niños, niñas y adolescentes}

\begin{tabular}{|c|c|c|}
\hline Subcategoría & Definición & Otros ejemplos \\
\hline \multirow[t]{2}{*}{ Ecociudadanía } & \multirow{2}{*}{$\begin{array}{l}\text { Desarrollo de competencias } \\
\text { proambientales de los } \\
\text { estudiantes }\end{array}$} & $\begin{array}{l}\text { "Tratar de hacer un grupo ambiental todos los } \\
\text { días de clases y en lo que están en clases salir } \\
\text { afuera a limpiar, recoger cajas o papeles, etc.» } \\
(\mathrm{G} 1, \mathrm{E} 6)\end{array}$ \\
\hline & & $\begin{array}{l}\text { «Existen buenas ideas de los forjadores } \\
\text { ambientales» }(\mathrm{G} 2, \mathrm{E} 6)\end{array}$ \\
\hline \multirow{2}{*}{$\begin{array}{l}\text { Enverdecimiento de la } \\
\text { escuela }\end{array}$} & \multirow{2}{*}{$\begin{array}{l}\text { Acciones emprendidas por parte } \\
\text { de la escuela con el fin de } \\
\text { producir plantas, árboles y todo } \\
\text { tipo de unidad vegetal }\end{array}$} & «Hay plantas medicinales» (G1, E4) \\
\hline & & «Cultivamos árboles nativos» (G1, E4) \\
\hline \multirow{2}{*}{$\begin{array}{l}\text { Ambientalización } \\
\text { institucional }\end{array}$} & \multirow{2}{*}{$\begin{array}{l}\text { Inclusión de la temática } \\
\text { ambiental en el curricullum o PIE }\end{array}$} & «Hacen talleres ambientales de $5^{\circ}$ a $8^{\circ} »(\mathrm{G} 1, \mathrm{E} 1)$ \\
\hline & & «Hay forjadores ambientales» (G4, E3) \\
\hline \multirow[b]{2}{*}{ Sensibilización territorial } & \multirow{2}{*}{$\begin{array}{l}\text { Acciones destinadas a la sensibi- } \\
\text { lización de las comunidades } \\
\text { contiguas o la sensibilización de } \\
\text { los estudiantes con su territorio } \\
\text { y otras realidades/ escuelas }\end{array}$} & «Que fuimos a Arauco» (G3, E2) \\
\hline & & $\begin{array}{l}\text { «Celebramos el Día del medioambiente y el } \\
\text { Día del agua» (G1, E4) }\end{array}$ \\
\hline \multirow{3}{*}{$\begin{array}{l}\text { Instalación sistema de } \\
\text { gestión de residuos }\end{array}$} & \multirow{3}{*}{$\begin{array}{l}\text { Instalación del sistema de } \\
\text { residuos requerido para la } \\
\text { certificación ambiental }\end{array}$} & «Tenemos punto limpio» $(G 2, E 2)$ \\
\hline & & $\begin{array}{l}\text { «Hay basureros de reciclaje en cada sala» (G1, } \\
\text { E4) }\end{array}$ \\
\hline & & $\begin{array}{l}\text { «Limpiamos la escuela y reciclamos } \\
\text { plástico» (G1, E4) }\end{array}$ \\
\hline
\end{tabular}

Uno de los aspectos que busca promover el Pipes es la ecociudadanía, lo que es destacado por los y las estudiantes sobre todo en las acciones de difusión, limpieza y cuidado medioambiental: «Hicimos carteles en taller de medio ambiente» (G1, E5); «limpiamos los patios en las sesiones del taller» (G1, E5). Se señala que las escuelas y la municipalidad promueven la capacidad de cuidado de las plantas y árboles, así como también el desarrollo de actividades ecológicas en general y se reitera la importancia de los forjadores ambientales, figura instalada por el programa del mismo nombre, proveniente del Ministerio del Medioambiente. ${ }^{6}$

\footnotetext{
6 Para mayor información sobre los forjadores ambientales visitar la página del Ministerio del Medioambiente http://educacion.mma.gob.cl/forjadores-ambientales/
} 
Otro objetivo fundamental del Pipes es lograr un paulatino enverdecimiento de la escuela, es decir, aumentar la masa verde presente. Aquí, los y las estudiantes se ven cautivados por los árboles nativos y las plantas medicinales, observándolos tanto dentro como fuera de la escuela, en espacios exclusivos y en estructuras de juego (por ejemplo, ruedas). Destacan cosas como que en sus establecimientos «se pueden obtener semillas» $\left(\mathrm{G}_{2}, \mathrm{E}_{4}\right)$ y que existe un «trencito de las plantas» $\left(\mathrm{G}_{3}, \mathrm{E}_{5}\right)$.

La ambientalización institucional, por su parte, refiere al grado de inclusión de la temática ambiental en los currículos y programas institucionales de la escuela. Esto lo aprecian en la contratación de un docente, la existencia de un taller ambiental, la obtención de sellos y certificaciones medioambientales, así como la presencia de otros programas ambientales en el establecimiento. Uno de los estudiantes, por ejemplo, mencionó como algo positivo el que «tenemos sello de reciclaje en la escuela» (G2, E2).

La sensibilización ambiental territorial está relacionada con las acciones del programa que trascienden la escuela o cuando se educa en ella a partir de recursos y saberes del territorio. Los y las estudiantes destacan dos tipos de acciones: las visitas y los eventos comunales. Las primeras son generalmente de tipo educativo, a otras escuelas o a sitios de interés como «visitar el parque eólico [Cuel]» (G2, E1), mientras que las segundas son eventos ya arraigados en el calendario comunal como el Día del Medioambiente y el Agua, la Feria de la Educación para la Sustentabilidad o cuando participan «en el Carnaval del reciclaje» (G1, E1).

El último activo está relacionado con el logro de uno de los indicadores del Sistema Nacional de Certificación de Establecimientos Educacionales: la instalación de un sistema de gestión de residuos sustentable en la escuela. Los y las estudiantes señalan la existencia de basureros y puntos limpios, siendo los primeros en muchas ocasiones diferenciados y orientados a reciclar materiales como el tetrapak, el papel, el cartón y el plástico. Destacan también la variedad de espacios utilizados con este fin, destacándose, por ejemplo, que sus escuelas «tienen basureros en el patio y salas de clases» $\left(G_{4}, E_{1}\right)$ o en el exterior de la escuela.

\section{Vacíos}

Otra categoría refiere a los vacíos, entendidos como ausencias de un aspecto importante en la escuela, vinculados con la temática ambiental y no considerado en el Pipes. Son elementos que a los estudiantes les gustaría que estuvieran presentes o que se mejora- 
ran en la escuela, pero que esta no ha abordado. Encontramos cuatro subcategorías: zonas recreativas, servicios higiénicos, alimentación insalubre y energías reutilizables (tabla 4).

\section{Tabla 4 \\ Vacíos identificados por niños, niñas y adolescentes}

\begin{tabular}{lll}
\hline \multicolumn{1}{c}{ Subcategoría } & \multicolumn{1}{c}{ Definición } & \multicolumn{1}{c}{ Otros ejemplos } \\
\hline $\begin{array}{l}\text { Zonas recreativas (cancha } \\
\text { de pasto, áreas verdes y } \\
\text { juegos recreativos) }\end{array}$ & $\begin{array}{l}\text { Espacio físico usado con fines } \\
\text { lúdicos o deportivos por los NNA }\end{array}$ & $\begin{array}{l}\text { "Que cuando llueve se hace poza ya que } \\
\text { las canchan son de tierra y de cemento y } \\
\text { que los arcos no tienen malla» (G3, E1) } \\
\text { «No hay césped ni flores» (G4, E3) }\end{array}$ \\
$\begin{array}{l}\text { Servicios higiénicos } \\
\text { (baños y fosas) }\end{array}$ & $\begin{array}{l}\text { Problema higiénico de gran } \\
\text { persistencia que no ha sido } \\
\text { atendido por la escuela }\end{array}$ & $\begin{array}{l}\text { «Un urinario esta malo en el baño» (G1, E4) } \\
\text { «El olor proveniente de las fosas» (G3, E6) }\end{array}$ \\
$\begin{array}{ll}\text { Alimentación insalubre } \\
\text { (chatarra, salada) }\end{array}$ & $\begin{array}{l}\text { Modelo de alimentación insalubre } \\
\text { que repercute en lo que y como } \\
\text { se come }\end{array}$ & "Que la comida tengo menos sal» (G3, E2) \\
$\begin{array}{l}\text { Energías reutilizables } \\
\text { (paneles solares) }\end{array}$ & $\begin{array}{l}\text { Tecnologías de energía reutiliza- } \\
\text { ble que no se encuentran instala- } \\
\text { das en la escuela }\end{array}$ & $\begin{array}{l}\text { «Nos gustaría que la escuela tuviera } \\
\text { paneles solares» (G1, E2) }\end{array}$ \\
\hline
\end{tabular}

Los y las estudiantes plantean que les gustarían más lugares donde realizar deporte, que los existentes tuvieran mejores condiciones y que la escuela creáse espacios nuevos donde divertirse y compartir: «Faltan más juegos recreativos» (G3, E2); «queremos más rincones verdes» $\left(\mathrm{G}_{1}, \mathrm{E}_{3}\right)$. Así, destacan como zonas recreativas las áreas verdes —árboles y plantas-, los juegos y las canchas. Señalan también estar aburridos de aquellas canchas de cemento o tierra, prefiriendo las que son de pasto, debido en gran parte a las lluvias que caen en la zona.

Otros problemas se refieren a los servicios higiénicos y la alimentación. En el primer caso, se alude a su baja cantidad y calidad, por ejemplo, señalando que «por mal cuidado se suben las fosas [del baño]» (G3, E5); en el segundo, se menciona su aporte nutricional. Al respecto, un niño comenta que le gustaría «que el kiosco no tuviera comida chatarra» $\left(G_{3}, E_{2}\right)$. Por último, menciona como vacío la falta de fuentes de energía renovable como los paneles solares. 


\section{Buenas prácticas}

Otra categoría que hace referencia a aspectos que no están comprendidos en el Pipes son las buenas prácticas, las cuales se definen como acciones proambientales provenientes de la escuela. Son consideradas buenas prácticas en la medida que los NNA ven en ellas un efecto positivo sobre su establecimiento. Entre las subcategorías presentes encontramos: el desarrollo de infraestructura; la limpieza y embellecimiento de la escuela; las acciones de $4 \mathrm{R}$; $\mathrm{y}$, por último, otras acciones proambientales (tabla 5 ).

\section{Tabla 5}

Buenas prácticas identificadas por niños, niñas y adolescentes

\begin{tabular}{|c|c|c|}
\hline Subcategoría & Definición & Otros ejemplos \\
\hline \multirow{3}{*}{$\begin{array}{l}\text { Desarrollo de } \\
\text { infraestructura }\end{array}$} & \multirow{3}{*}{$\begin{array}{l}\text { Creación de nueva infraestruc- } \\
\text { tura por parte de la escuela y la } \\
\text { comunidad escolar }\end{array}$} & $\begin{array}{l}\text { «De vuelta de vacaciones de invierno } \\
\text { confeccionamos un invernadero.» (G1, E1) }\end{array}$ \\
\hline & & «Tenemos un laboratorio natural» (G1, E4) \\
\hline & & «Tenemos compostera» $(\mathrm{G} 3, \mathrm{E} 6)$ \\
\hline \multirow{3}{*}{$\begin{array}{l}\text { Limpieza y embelleci- } \\
\text { miento de la escuela }\end{array}$} & \multirow{3}{*}{$\begin{array}{l}\text { Acciones de aseo, ornato y } \\
\text { estética provenientes de la } \\
\text { comunidad escolar }\end{array}$} & $\begin{array}{l}\text { «Tenemos inspectores que barren nuestro } \\
\text { patio» (G1, E1) }\end{array}$ \\
\hline & & «Ponerles ropa a los árboles» (G2, E5) \\
\hline & & $\begin{array}{l}\text { «Lo bueno es que se preocupan de que esté } \\
\text { limpio el patio» (G2, E6) }\end{array}$ \\
\hline \multirow{2}{*}{$\begin{array}{l}\text { Reducir, reutilizar, } \\
\text { reciclar y recuperar }\end{array}$} & \multirow{2}{*}{$\begin{array}{l}\text { Acciones de las } 4 \mathrm{R} \text { realizadas } \\
\text { por la comunidad escolar }\end{array}$} & $\begin{array}{l}\text { «Creamos abono para plantas, cultivamos y } \\
\text { replantamos» }(\mathrm{G} 3, \mathrm{E} 6)\end{array}$ \\
\hline & & «Hacemos eco ladrillos» (G3, E6) \\
\hline \multirow{2}{*}{$\begin{array}{l}\text { Otras acciones } \\
\text { proambientales }\end{array}$} & \multirow{2}{*}{$\begin{array}{l}\text { Acciones directas de cuidado } \\
\text { del entorno provenientes de la } \\
\text { comunidad escolar }\end{array}$} & «Existe la patrulla araucaria» $(\mathrm{G} 4, \mathrm{E} 3)$ \\
\hline & & $\begin{array}{l}\text { «Que se puede salvar a los animales en } \\
\text { peligro de extinción» }(G 3, E 4)\end{array}$ \\
\hline
\end{tabular}

El desarrollo de infraestructura se refiere a la creación de nuevas estructuras construidas por la escuela con el fin de mejorar las condiciones hasta ese entonces presentes. Los y las menores destacan la creación y mejoramiento tanto de espacios como de estructuras particulares. De esta manera, son mencionados: el invernadero, la compostera, los viveros, los centros de acopio, los laboratorios naturales, las áreas verdes, etc. En muchas ocasiones la preocupación ambiental de las escuelas hace que desarrollen más de una de estas, como menciona el siguiente estudiante: «lo bueno es que tenemos un vivero, un centro de acopio y nos preocupamos por el medioambiente» (G2, E6). 
La segunda subcategoría refiere a la limpieza y el embellecimiento del establecimiento, incluyendo aquí toda acción orientada a volver la escuela un espacio más ameno, limpio y estético. Aquí los menores son capaces de detectar la importancia de los auxiliares de la educación y su fundamental rol en el mantenimiento de la escuela; a su vez, destacan repetidamente su propio aporte en esta función: «cada vez que podemos limpiamos los tambores de reciclaje» (G1, E5). Por último, son varias veces destacadas las acciones de embellecimiento, tales como colocar ropa a los árboles o pintar objetos de la escuela.

Por su parte, las llamadas acciones de las ${ }_{4} \mathrm{R}$ comprenden toda reducción, reutilización, reciclaje y recuperación de los desperdicios que genera la escuela. Aquí se señala fuertemente la utilización de los desperdicios orgánicos e inorgánicos, siendo actividades relevantes en el discurso de los y las estudiantes la producción de ecoladrillos y la generación de abono. En este contexto destacan en general las actividades educativas «donde se utilizan objetos reciclables» $\left(G_{4}, E 1\right)$. La última subcategoría incluye a todas aquellas acciones no contenidas en las anteriores, pero que están relacionadas con la protección del medioambiente, la escuela y la convivencia interna, yendo desde «no pelear» $\left(\mathrm{G}_{1}, \mathrm{E}_{3}\right)$ hasta mantener «vivos los gusanos» $\left(\mathrm{G}_{3}, \mathrm{E}_{6}\right)$.

\section{Problemáticas socioambientales}

La última categoría se refiere a problemáticas socioambientales percibidas en su territorio o en cualquier latitud del planeta. Aparecen en el discurso de los menores como acciones lamentables que realiza la especie humana y que emergieron al hablar de los aspectos negativos. Aquí agrupamos dos subcategorías: conducta antrópica y desperdicio de los recursos (tabla 6).

La primera es entendida como aquellas acciones que, producto de la intención humana, terminan por dañar la naturaleza, siendo clave en la formulación de los NNA el asociar este daño a una acción particular de una o más personas. Esta acción es generalmente realizada por un «otro distinto a mí», con notoria excepción en la falta de cuidado y ensuciamiento de los espacios comunes de la escuela, acción que realizaría la mayoría de los y las estudiantes. Esto se diferencia de la primera categoría, en que el foco de lo dicho está en la acción de daño y no en la falta de competencias o de hábito de sus compañeros, así como tampoco en la falta de desarrollo del programa en la escuela.

Los y las NNA del estudio atribuyen el daño a tres grandes «responsables»: las personas, las escuelas y las industrias. Las primeras son descritas como descuidadas con la naturaleza, lo que se refleja en el maltrato de los animales, de la flora y en la mala utili- 
zación del agua: «Se suben a los árboles, sacan y pisan las plantas» (G3, E5). Las segundas son responsabilizadas del mal cuidado de los árboles y vegetales, mientras que las terceras son asociadas al humo y la contaminación ambiental: «Que quemen los bosques y maten a los animales o los dejen sin hogar» (G2, $\left.\mathrm{E}_{4}\right)$.

\section{Tabla 6}

\section{Problemáticas socioambientales detectadas por niños, niñas y adolescentes}

\begin{tabular}{|c|c|c|}
\hline Subcategoría & Definición & Otros ejemplos \\
\hline \multirow{3}{*}{$\begin{array}{l}\text { Conducta antrópica } \\
\text { (NNA, personas, } \\
\text { industrias) }\end{array}$} & \multirow{3}{*}{$\begin{array}{l}\text { Acción impulsada por una persona } \\
\text { o conjunto de estas que daña de } \\
\text { manera directa la naturaleza o los } \\
\text { recursos }\end{array}$} & $\begin{array}{l}\text { «Destruyen los árboles, las plantas y las } \\
\text { flores» }(G 4, E 3)\end{array}$ \\
\hline & & $\begin{array}{l}\text { "Que botan basura y a veces botan perros } \\
\text { en el camino» }(G 3, E 4)\end{array}$ \\
\hline & & $\begin{array}{l}\text { «Que las industrias no dejan de tirar humo } \\
\text { y contaminan el medioambiente» }(\mathrm{G} 3, \mathrm{E} 4)\end{array}$ \\
\hline \multirow{2}{*}{$\begin{array}{l}\text { Desperdicio de los recur- } \\
\text { sos (agua, materiales, } \\
\text { comida, luz) }\end{array}$} & \multirow{2}{*}{$\begin{array}{l}\text { Mal utilización de un recurso tanto } \\
\text { natural como industrial producto de } \\
\text { la acción humana }\end{array}$} & «No utilizan bien el papel» $(\mathrm{G} 4, \mathrm{E} 3)$ \\
\hline & & $\begin{array}{l}\text { «Destruyen los materiales y los botan al } \\
\text { suelo» }(G 4, E 3)\end{array}$ \\
\hline
\end{tabular}

Los y las estudiantes describen variadas maneras de causar daño antrópico, siendo utilizadas una serie de palabras tales como destruir, romper, quebrar, subirse, colgarse, rayar, pisar y arrancar. Distinguen también aquello que se daña: seres vivos, como animales y árboles; espacios, como el patio, parques y bosques; y también cosas, como materiales e infraestructura de la escuela.

La segunda subcategoría refiere a aquellas acciones humanas, no necesariamente intencionales, que detraen en la mala utilización de recursos naturales o industriales. Los NNA identifican así el desperdicio del agua, luz y comida, como también el mal aprovechamiento de recursos que provienen de la escuela. Remarcan la importancia de no dejar el agua de las cañerías corriendo. En general sostienen «que las personas no cuidan el agua» (G2, E4) y que «desperdician la comida» (G1, E1).

\section{Discusión}

En la presente investigación se preguntó por las opiniones y percepciones de un grupo de escolares sobre un programa de educación ambienta local del que forman parte. Se observa que son capaces de referir tanto a actividades en que participaron directamente 
como a aspectos internos del programa (objetivos, estructura, estrategias y gestión) y externos (condiciones de implementación, contexto de cultura y estructura escolar, relación con otros programas), incluyendo opiniones sobre problemáticas socioambientales locales y globales. Demuestran con ello una capacidad crítica de evaluación del programa en relación con los aprendizajes individuales, colectivos e institucionales que este busca y logra mejorar. En este sentido, presentan una serie de percepciones sobre cómo se desarrolla - o cómo se debería desarrollar - la temática ambiental en su escuela o comunidad, habilidad que ya había sido constatada en anteriores estudios con escolares y pre-escolares (Ceballos-López \& García-Becerra, 2018; Woolner et al., 2007).

A través de sus percepciones identificamos aspectos negativos y positivos de la implementación de un PEA local y de su entorno. Según sus voces tres aspectos son negativos: 1) dificultades en el logro de los objetivos; 2) vacíos; 3) problemáticas socioambientales. Así mismo, dos aspectos son positivos en la implementación del programa: 1) los activos del programa; $y, 2$ ) las buenas prácticas ambientales desarrolladas en la escuela.

Los NNA fueron capaces de detectar cuando las metas del programa están lejos de cumplirse, pues todas las dificultades señaladas están relacionadas con los objetivos explícitos de los programas ambientales que se instalan en la escuela. Destacan también cuando lo que sucede es lo contrario, mencionando aquellos activos que facilitan la efectividad del programa en el cumplimiento de sus objetivos. Para ambos casos mencionan elementos relacionados con la ecociudadanía, con la ambientalización institucional y territorial, con el enverdecimiento de la escuela y con la instalación de un sistema sustentable de gestión de residuos escolares.

En síntesis, las mejoras extraíbles de la voz del alumnado apuntan a estrategias de gestión de recursos materiales (residuos, producción vegetal) y subjetivos (compromiso, competencias proambientales), lo que sugiere fortalecer técnicas de difusión y actividades de resultados visibles y continuos (por ejemplo: limpieza, cuidado, plantación). Otra gestión importante que se deriva es la de redes externas (con otros programas ministeriales o en visitas al territorio) e internas (articulación con el currículo). Además, se amplían los alcances del programa a nuevas posibles acciones de energía reutilizable (paneles solares), que vinculen la sensibilización ambiental con el bienestar del cuerpo (deporte, higiene, alimentación) y las relaciones sociales (recreación colectiva, zonas verdes).

Los resultados hacen pensar que son capaces de identificar cuándo la temática ambiental se desarrolla de manera correcta en su escuela, no significando con esto la absoluta conciencia sobre el objetivo implícito, sino más bien la impresión que genera en ellos 
cierto ámbito de desarrollo ambiental (Ceballos-López \& García-Becerra, 2018; García \& Moreno, 2015). De cierta manera son capaces también de aprehender la complejidad de estos programas, a la vez que dan detalles de sus particularidades.

En suma, estos resultados hablan de una aprehensión de la temática ambiental por parte de los y las estudiantes y de cierta mirada crítica en ellos que es posible plasmar en el discurso sobre cómo se desarrolla lo ambiental en su escuela y en su entorno (Liebel \& Gaitán, 2019; Rojas-Maturana \& Peña-Cortés, 2015). En este sentido, tal como en otras investigaciones similares (Ärlemalm-Hagsér, 2013; García \& Moreno, 2015; Imhoff \& Brussino, 2013) se pudo evidenciar que los NNA son sujetos activos en identificar las problemáticas ambientales, tanto locales como globales, demostrando cierta capacidad de pensamiento glocal. También - y en la misma línea de los citados estudios- los y las estudiantes demuestran facilidad para conversar sobre los temas ambientales, sus diversos actores, desafíos y dificultades, a fin de analizarlos críticamente.

Siguiendo la línea de los estudios de Susinos-Rada y Ceballos-López (2012) y Fielding (2011) fue posible identificar la intencionalidad de los y las estudiantes por participar cuando se les ofrecen las condiciones y cuando son verdaderamente escuchados. La mencionada evaluación municipal facilitó la inclusión de las demandas de los escolares en la programación correspondiente al presente año del programa (2019). La intencionalidad fue asumir la absoluta validez de estos sujetos sociales y de derecho como personas importantes en todas las fases de la construcción y desarrollo de los proyectos, sistemas y programas ambientales locales (Díaz, 2010; Díaz-Bórquez et al., 2018).

Esta misma valoración de su aptitud crítica se ve reforzada por su capacidad para identificar si lo implementado en su escuela responde a los verdaderos objetivos de aquel proyecto ambiental, utilizando para ello calificativos que aluden a la insuficiencia o déficit en un ámbito. Por tanto, incorporar esta visión es fundamental para la configuración de PEA cada vez más efectivos y eficientes. En este sentido, este estudio es un aporte tanto metodológico como empírico para posteriores programas.

Aun así, futuras investigaciones podrían utilizar metodologías que consideren otras fases para la participación del alumnado, pues así lograrían utilizar para estos fines categorías que les proporcionen más sentidos, siendo una limitación de este estudio que las categorías provengan desde los investigadores e investigadoras. A su vez, es posible pensar como una limitante el que no se midió de manera sistemática el rol y el protagonismo que como estudiantes desempeñan en el desarrollo del programa. Por último, como aprendizaje cabe consignar la importancia de utilizar registros de audios acuciosos que 
permitan aprehender tanto las voces individuales como colectivas que emergen en este tipo de instancias.

En este marco, es un desafío de toda producción científica y educativa que se pregunte por la participación con menores el avanzar hacia mejores y mayores grados de participación de estos. En este sentido, resultan útiles las taxonomías ofrecidas por autores como Hart (2013) o Trilla y Novella (2001), pues marcan un horizonte paulatino por el cual se debe transitar hacia una plena inclusión de los menores en la producción de conocimiento científico y en la gestión de proyectos de investigación y proyectos educativos tanto en EA como en otras disciplinas.

Así, uno de los desafíos que tiene la EA es empezar a configurar un campo disciplinar que considera las especificidades del trabajo con NNA y que, a la vez, atiende sus objetivos. En este marco, es importante entender la necesidad de la participación infantil bajo el lente de la actual crisis ambiental. Desarrollar, por tanto, una EA que sea pertinente a los estudiantes es un desafío a fin de involucrar de mejor manera a un importante actor social ante la presente crisis humanitaria.

Cabe pensar, entonces, cómo se incluyen los sentidos y significados que otorgan los y las estudiantes a la EA, como también aquellos aspectos que evalúan positiva o negativamente de la implementación de los PEA locales. En ese sentido, se empatiza con los proyectos promotores del desarrollo de conducta ambiental; sin embargo, se entiende que uno de los grandes desafíos de los proyectos ambientales es mejorar las condiciones contextuales e internas para su implementación. Así, pensado la participación infantil desde la óptica de este estudio, esta vuelve a ver reforzada su doble valía: por un lado, es el mandato ético a cumplir con los derechos sociales de los NNA; por otro, la indesmentible importancia de estos en el logro de los objetivos de dichos programas.

\section{Agradecimientos}

El estudio no contó con ninguna fuente de financiamiento externo. De todas maneras, corresponde agradecer las gestiones realizadas por la Dirección de Administración de Educación Municipal de la comuna de Los Ángeles en materias de coordinación, transporte y disposición de las condiciones necesarias para la realización de las técnicas de producción de la información. 


\section{Referencias}

Alonso, A. (2018, 28 de diciembre). Dirección de educación entrega PADEM a Concejo municipal. Municipalidad de Los Ángeles. http://www.losangeles.cl/w/?p=47805

Ärlemalm-Hagsér, E. (2013). Minds on Earth Hour-a theme for sustainability in Swedish early childhood education. Early Child Development and Care, 183(12), 1782-1795.

https://doi.org/10.1080/03004430.2012.746971

Ärlemalm-Hagsér, E., \& Davis, J. (2014). Examining the rhetoric: A comparison of how sustainability and young children's participation and agency are framed in Australian and Swedish early childhood education curricula. Contemporary Issues in Early Childhood, 15(3), 231-244. https://doi.org/10.2304/ciec.2014.15.3.231

Ceballos-López, N., \& García-Becerra, J. (2018). Los alumnos de $4^{\circ}$ de educación primaria como investigadores de su barrio. En Susinos, T., Ceballos, N., \& Saiz, Á. (Eds.), Cuando todos cuentan: experiencias de participación de estudiantes en las escuelas. Muralla.

Ceballos-López, N., Susinos-Rada, T., \& García-Lastra, M. (2019). Espacios para jugar, para aprender. Espacios para relacionarse. Una experiencia de voz del alumnado en la escuela infantil (o-3 años). Estudios Pedagógicos, 44(3), 117-135. http://dx.doi.org/ 10.4067/So718-07052018000300117

Clark, A. (2011). Breaking methodological boundaries? Exploring visual, participatory methods with adults and young children. European Early Childhood Education Research Journal, 19(3), 321-330. https://doi.org/10.1080/1350293X.2011.597964

Contreras, C., \& Pérez, A. (2011). Participación invisible: niñez y prácticas participativas emergentes. Revista Latinoamericana de Ciencias Sociales, Niñez y Juventud, 9(2), 811-825.

Díaz, S. (2010). Participar como niña o niño en el mundo social. Revista Latinoamericana de Ciencias Sociales, Niñez y Juventud, 8(2), 1149-1157.

Díaz-Bórquez, D., Contreras-Shats, N., \& Bozo-Carrillo, N. (2018). Participación infantil como aproximación a la democracia: desafíos de la experiencia chilena. Revista Latinoamericana de Ciencias Sociales, Niñez y Juventud, 16(1), 101-113. https://doi.org/ 10.11600/1692715X.16105

Dixson-Declève, S., \& Wijkman, A. (2019, 23 de enero). This could be the biggest scandal of the Climate Change Era. The Huffington Post. https://www.huffpost.com/entry/ climate-change-plan_n_5c41c704e4bobfa693c2agob

Escobar, J., \& Bonilla-Jimenez, F. (2017). Grupos focales: una guía conceptual y metodológica. Cuadernos hispanoamericanos de psicología, 9(1), 51-67. 
Escobedo, P., Sales, A., \& Traver-Martí, J. A. (2017). La voz del alumnado: su silencio y la cultura profesionalista. Educación XX1: Revista de la Facultad de Educación, 20(2), 299318. https://doi.org/10.5944/educxx1.19045

Espejel, A., \& Flores, A. (2017). Experiencias exitosas de educación ambiental en los jóvenes del bachillerato de Tlaxcala, México. Revista Luna Azul, (44). https://doi.org/ 10.17151/luaz.2017.44.18

Fielding, M. (2011). La voz del alumnado y la inclusión educativa: una aproximación democrática radical para el aprendizaje intergeneracional. Revista interuniversitaria de formación del profesorado, (70), 31-62.

Fielding, M., \& McGregor, J. (2005, abril 11-15). Deconstructing student voice: New spaces for dialogue or new opportunities for surveillance? [Presentación de artículo]. American Educational Research Association Annual Conference (AERA), Montreal, Canadá. http://www.leeds.ac.uk/educol/documents/155816.pdf

García, F., \& Moreno, O. (2015). De la educación ambiental a la educación ciudadana planetaria: alumnado y profesorado en el programa educativo Ecoescuela en Andalucía. Biblio 3W: Revista Bibliográfica de Geografía y Ciencias Sociales, 20(1124), 1-28.

González, E., \& Bonan, L. (2017). Saber no alcanza para actuar: revisión y reflexiones acerca de la relación entre el conocimiento y la adopción de conductas ambientales. Ciencia \& Educação, 23(2), 357-372. https://doi.org/10.1590/1516-731320170020005

González-Gaudiano, E., \& Maldonado-González, A. (2017). Amenazas y riesgos climáticos en poblaciones vulnerables: el papel de la educación en la resiliencia comunitaria. Teoría de la Educación, 29(1), 273-294. https://doi.org/10.14201/teoredu291273294

Hart, R. (2013). Children's participation: The theory and practice of involving young citizens in community development and environmental care. Routledge.

Hedefalk, M., Almqvist, J., \& Östman, L. (2015). Education for sustainable development in early childhood education: A review of the research literature. Environmental Education Research, 21(7), 975-990. https://doi.org/10.1080/13504622.2014.971716

Hidalgo, N., \& Perines, H. (2018). Dar voz a los protagonistas: la participación estudiantil en el proceso de enseñanza-aprendizaje. Revista Educación, 42(2), 438-464. https://doi.org/10.15517/revedu.v42i2.27567

Huckle, J. (1991). Education for sustainability: Assessing pathways to the future. Australian Journal of Environmental Education, 7, 43-62. https://doi.org/10.1017/So814062600001853 Imhoff, D., \& Brussino, S. (2013). Participación sociopolítica infantil y procesos de socialización política: exploración con niños y niñas de la ciudad de Córdoba, Argentina. Liberabit, $19(2), 205^{-213}$. 
Jensen, B. (2004). Environmental and health education viewed from an action-oriented perspective: A case from Denmark. Journal of Curriculum Studies, 36(4), 405-425. https://doi.org/10.1080/0022027032000167235

Liebel, M. (2007). Paternalism, participation and children's protagonism. Children Youth and Environments, $17(2), 56-73$.

Liebel, M., \& Gaitán, L. (2019). El poder de los niños y niñas: anotaciones sobre el protagonismo de movimientos infantiles en la actualidad. Sociedad e Infancias, 3, 15-20. https://doi.org/10.5209/soci.65352

Mackey, G. (2012). To know, to decide, to act: The young child's right to participate in action for the environment. Environmental Education Research, 18(4), 473-484. https://doi.org/10.1080/13504622.2011.634494

Martínez-Agut, M., Ull, M., \& Aznar-Minguet, P. (2014). Education for sustainable development in early childhood education in Spain: Evolution, trends and proposals. European Early Childhood Education Research Journal, 22(2), 213-228. https://doi.org/ 10.1080/1350293X.2013.783299

Medina, I., \& Páramo, P. (2014). Research in environmental education in Latin America: A bibliometric analysis. Revista Colombiana de Educación, (66), 55-72.

Mitra, D. (2007). Student voice in school reform: From listening to leadership. En D. Thiessen \& A. Cook-Sather (Eds), International handbook of student experience in elementary and secondary school (pp. 727-744). Springer. https://doi.org/10.1007/1-4020-3367-2_29

Mogren, A., Gericke, N., \& Scherp, H. Å. (2018). Whole school approaches to education for sustainable development: A model that links to school improvement. Environmental Education Research, 24(4), 508-531. https://doi.org/10.1080/13504622.2018.1455074

Municipalidad de Los Ángeles. (2016). Ordenanza ambiental en la comuna de Los Ángeles. Departamento de Medioambiente.

Muñoz-Montilla, A., \& Páramo-Bernal, P. (2018). Monitoreo de los procesos de educación ambiental: propuesta de estructuración de un sistema de indicadores de educación ambiental. Revista Colombiana de Educación, (74), 81-106. https://doi.org/ 10.17227/rce.num74-6899

Navas, M., Victoria, M., Martínez, M., \& Valdebenito, X. (2018). Marcos éticos para la investigación en educación con población infantil y juvenil: hacia una propuesta de orientaciones. Centro de Estudios Mineduc.

Olsson, D., Gericke, N., \& Chang, S. N. (2016). The effect of implementation of education for sustainable development in Swedish compulsory schools-assessing pupils' 
sustainability consciousness. Environmental Education Research, 22(2), 176-202. https://doi.org/10.1080/13504622.2015.1005057

Olsson, D., Gericke, N., Boeve-de Pauw, J., Berglund, T., \& Chang, T. (2019). Green schools in Taiwan-Effects on student sustainability consciousness. Global Environmental Change, 54, 184-194. https://doi.org/10.1016/j.gloenvcha.2018.11.011

Patton, M. (1990). Qualitative evaluation and research methods. Sage.

Peters, D., Adam, T., Alonge, O., Agyepong, I., \& Tran, N. (2013). Implementation research: What it is and how to do it. BMJ, 347, f6753. https://doi.org/10.1136/bmj.f6753

Pramling-Samuelsson, I. (2011). Why we should begin early with ESD: The role of early childhood education. International Journal of Early Childhood, 43(2), 103-118. https://doi.org/10.1007/s13158-011-0034-x

Rojas-Maturana, M., \& Peña-Cortés, F. (2015). Saberes ambientales lafkenche en escuelas de la costa de La Araucanía (Chile). Revista Latinoamericana de Ciencias Sociales, Niñez y Juventud, 13(2), 1207-1220. https://doi.org/10.11600/1692715x.13245171214

Rudduck, J., \& Flutter, J. (2007). Cómo mejorar tu centro escolar dando la voz al alumnado. Morata.

Saidón, M., \& Claverie, J. (2016). Percepciones de docentes y directores sobre los factores que promueven u obstaculizan la educación ambiental en escuelas del Área Metropolitana de Buenos Aires. Ciência \& Educação, 22(4), 993-1012. http://dx.doi.org/ 10.1590/1516-731320160040010

Sauvé, L. (2014). Educación ambiental y ecociudadanía: dimensiones claves de un proyecto político-pedagógico. Revista científica, 1(18), 12-23. https://doi.org/10.1136/ bmj.f6753

Susinos-Rada, T., \& Ceballos-López, N. (2012). Voz del alumnado y presencia participativa en la vida escolar: apuntes para una cartografía de la voz del alumnado en la mejora educativa. Revista de Educación, 359, 24-44. http://dx.doi.org/10.4438/1988-592XRE-2012-359-194

Trilla, J., \& Novella-Cámara, A. M. (2001). Educación y participación social de la infancia. Revista Iberoamericana de Educación (OEI), 26, 137-164.

Vaismoradi, M., Turunen, H., \& Bondas, T. (2013). Content analysis and thematic analysis: Implications for conducting a qualitative descriptive study. Nursing \& Health Sciences, 15(3), 398-405. https://doi.org/10.1111/nhs.12048

Voltarelli, M. (2018). Los temas del protagonismo y la participación infantil en las producciones sudamericanas. Revista Latinoamericana de Ciencias Sociales, Ninez y Juventud, 16(2), 741-756. https://doi.org/10.1160o/1692715x.16207 
Winkler, M. I., Alvear, K., Olivares, B., \& Pasmanik, D. (2014). Psicología comunitaria hoy: orientaciones éticas para la acción. Psicoperspectivas, 13(2), 43-54. http://dx.doi.org/ 10.5027/psicoperspectivas-Vol13-Issuez-fulltext-353

Woolner, P., Hall, E., Wall, K., \& Dennison, D. (2007). Getting together to improve the school environment: User consultation, participatory design and student voice. Improving Schools, 10(3), 233-248. https://doi.org/10.1177/1365480207077846

World Economic Forum. (2019, 25 de enero). The Global Risks Report 2019. https:// www.weforum.org/reports/the-global-risks-report-2019 